\title{
Kurzbeiträge
}

Nervenarzt 2022 $\cdot 93: 175-178$

https://doi.org/10.1007/s00115-021-01124-x

Angenommen: 15. März 2021

Online publiziert: 30. April 2021

(c) Der/die Autor(en) 2021

\author{
Wilhelm Haverkamp ${ }^{1,3}$. Olaf Göing ${ }^{2}$ - Markus Anker ${ }^{1,3} \cdot$ Stefan D. Anker ${ }^{1,3}$ \\ ${ }^{1}$ Abteilung für Kardiologie und Metabolismus, Med. Klinik für Kardiologie, Campus Virchow-Klinikum, \\ Charité - Universitätsmedizin Berlin, Berlin, Deutschland \\ ${ }^{2}$ Sana Klinikum Lichtenberg, Berlin, Deutschland \\ ${ }^{3}$ Berlin Institute of Health Center for Regenerative Therapies (BCRT), Berlin, Deutschland
}

\section{Vorhofflimmerndiagnostik mittels EKG-fähiger Smartwatches}

lyse. Wenn sich hierbei der Verdacht auf Vorhofflimmern ergibt, wird der Patient via App benachrichtigt und gebeten, ein EKG zu registrieren. Die bislang zur Validierung dieser neuen Techniken zur Verfügung stehenden Daten reichen für eine endgültige Bewertung ihrer diagnostischen Wertigkeit unter Real-worldBedingungen nicht aus [1]. Im Vorfeld $\mathrm{zu}$ erwartender Studienergebnisse erschien es den Autoren dieses Beitrages sinnvoll, die Algorithmen und Arbeitsweisen EKG-fähiger Smartwatches zu analysieren (•Tab. 1). Hierzu wurden die im Rahmen von Zulassungsverfahren und der Bewerbung öffentlich gemach- ten Informationen zur EKG-Funktion ausgewertet [2-6]. An diese Informationen heranzukommen, erwies sich zum Teil als sehr aufwendig. Die Politik ist gefordert, Standards zu schaffen, die es Ärzten erlaubt, neue Techniken (die als Medizinprodukte zugelassen sind) unter medizinischen Gesichtspunkten realistisch bewerten zu können.

\section{Diagnostische Genauigkeit}

Wenn es um die reine Erkennung von Vorhofflimmern geht, liegen die für EKG-fähige Smartwatches mitgeteilten Sensitivitäten und Spezifitäten bei über vorausgesetzt) einer dauerhaften oralen Antikoagulation zuzuführen [1]. In diesem Zusammenhang ist wichtig, dass ein mittels Smartwatch-EKG dokumentiertes, $30 \mathrm{~s}$ anhaltendes Vorhofflimmern (die Registrierdauer beträgt max. $30 \mathrm{~s}$ ) laut aktueller Leitlinien dann diagnostisch verwertbar ist, wenn die Diagnose von einem Arzt bestätigt wurde - eine erneute Bestätigung der Diagnose durch ein weiteres EKG-Verfahren (z. B. ein Langzeit-EKG) ist nicht notwendig [1].

\section{Algorithmen und Arbeitsweise}

EKG-fähige Smartwatches sind mit Software-Applikationen (Apps) ausgestattet, die es erlauben, Vorhofflimmern automatisch $\mathrm{zu}$ diagnostizieren. Andere Rhythmusstörungen werden nicht automatisch erkannt. Ein Teil der Smartwatches ermöglicht darüber hinaus ein Vorhofflimmernscreening mittels photoplethysmographischer Pulswellenana- 


\section{Vorhofflimmern - 122 BPM $\varnothing$}

Dieses EKG deutet auf Vorhofflimmern und eine hohe Herzfrequenz hin.

Wenn du dieses Ergebnis nicht erwartet hast oder deine Herzfrequenz hoch bleibt, solltest du bald mit deinem Arzt sprechen

\section{Gemeldete Symptome}

- Schneller, hämmernder oder flatternder Herzschlag

- Übersprungener Herzschlag

- Kurzatmigkeit
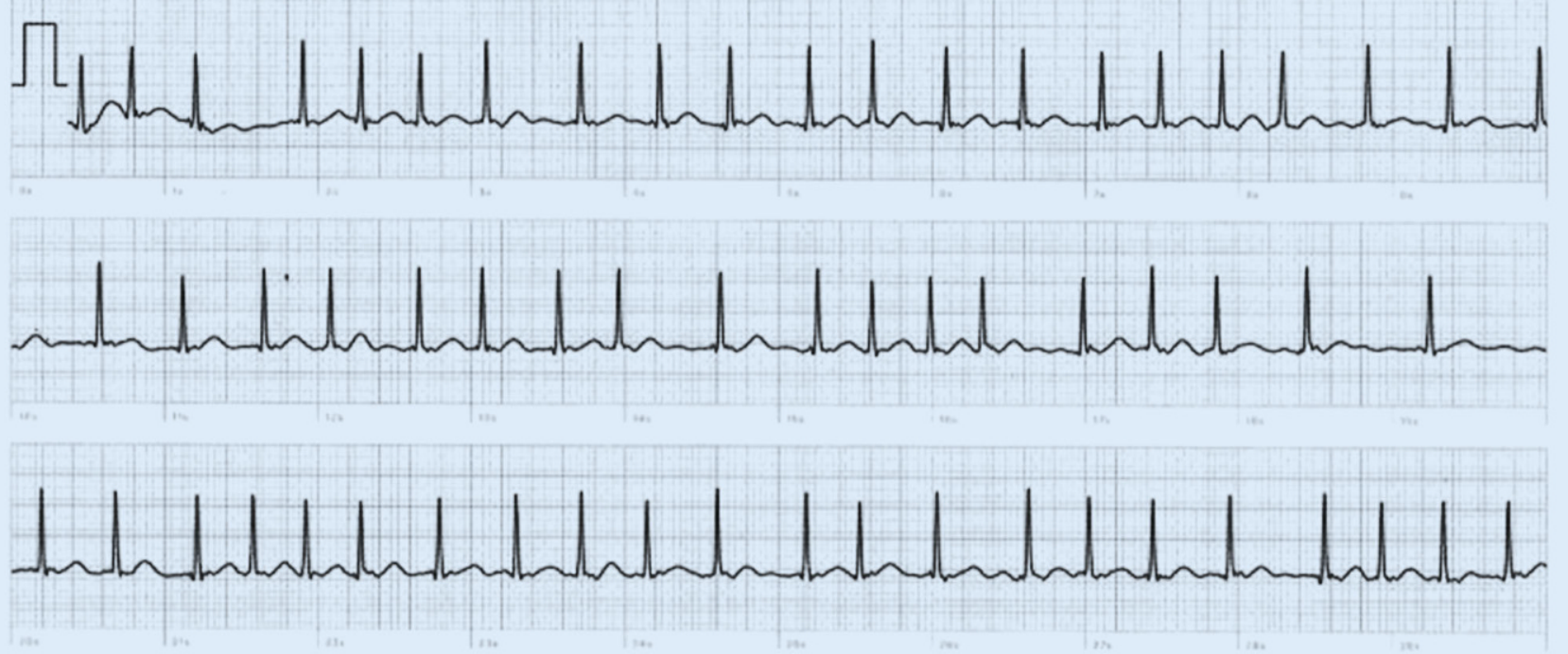

Abb. 1 ॥ 56-jähriger Patient. Der automatische Algorithmus diagnostiziert Vorhofflimmern, Kammerfrequenz 122/min.

Apple Watch Series 5

95\% [2-7]. Anzumerken ist, dass die Untersuchungen unter sehr standardisierten Bedingungen stattfanden. Darüber hinaus beziehen sich diese Angaben auf auswertbare EKGs - etwa 10-20\% der mit einer Smartwatch registrierten EKGs sind nicht auswertbar (z.B. bedingt durch Artefakte). Die in - Tab. 1 zusammengestellten Daten deuten drauf hin, dass die diagnostische Genauigkeit im Alltag niedriger sein dürfte. Vorhofflimmern mit einer Kammerfrequenz unterhalb von 50/min wird von keiner der Smartwatches erkannt. Auch bei hohen Herzfrequenzen ergibt sich ein Grenzwert, ab dem nicht mehr auf Vorhofflimmern geprüft wird (• Abb. 1 und 2). Dieser liegt bei den meisten Uhren bei $120 / \mathrm{min}$. Eine überarbeitete Version des Apple-Algorithmus detektiert jetzt auch Vorhofflimmern mit einer Kammerfrequenz von bis $\mathrm{zu} 150 / \mathrm{min}$ [7]. Die Genauigkeit des Algorithmus ist jedoch bei Kammerfrequenzen von über 100/min deutlich niedriger als bei einer Kammerfrequenz von $50-99 / \mathrm{min}$
(98,3\% gegenüber $83 \%$; [7]). In diesem Zusammenhang ist es wichtig festzustellen, dass neu auftretendes Vorhofflimmern unter klinischen Bedingungen in etwa einem Drittel der Fälle Kammerfrequenzen von über 120/min aufweist, nicht selten liegt die Frequenz über 150/min [8].

Beim Smartwatch-basierten Screening auf Vorhofflimmern muss berücksichtigt werden, dass die hierbei genutzten photoplethysmographischen Messungen - da sie viel Strom verbrauchen - nur in Ruhe und nur intermittierend erfolgen. Das Vorgehen unterscheidet sich herstellerabhängig. Es entstehen diagnostische Lücken, die dazu führen, dass insbesondere kurze Arrhythmieepisoden nur unzureichend erkannt werden. Für solche diagnostische Lücken spricht auch die relativ geringe Anzahl an Benachrichtigungen über Vorhofflimmern in der AppleHeart-Studie, in die mehr als 400.000 Personen eingeschlossen wurden [9]. Eine Benachrichtigung über Pulsunre- gelmäßigkeiten via App erfolgte nur bei $0,52 \%$ der Probanden. Mit 3,2\% war die Benachrichtigungsrate auch in der kleinen Subgruppe der über 65-Jährigen $(n=24,6326)$ relativ niedrig. Die Studie hat gezeigt, dass ein Smartwatchbasiertes Screening auf Vorhofflimmern prinzipiell möglich ist, die diagnostische Genauigkeit des Verfahrens lässt sich aber, da eine Positivkontrolle fehlte, nicht berechnen. Studien an kleinen Kollektiven, die eine relativ hohe Sensitivität zeigen, reichen für eine abschließende Bewertung nicht aus [10].

\section{Konsequenzen für den Alltag}

Basierend auf den derzeit implementierten, je nach Hersteller unterschiedlichen Algorithmen bzw. Arbeitsweisen von Smartwatches bestehen, wie auch bei allen anderen EKG-Verfahren, Lücken bei der Diagnostik von Vorhofflimmern. Diese sollten dem Anwender und Auswerter von Smartwatch-EKGs bekannt sein. Eine Konsequenz ist z. B., dass 
Herzfrequenz ist über $150-\vee 161 \mathrm{BPM} \varnothing$

Dieses EKG wurde nicht auf Vorhofflimmern geprüft, da eine Herzfrequenz über 150 BPM betrug.

\section{Gemeldete Symptome}

•Schneller, hämmernder oder flatternder Herzschlag

Wenn dieses Ergebnis wiederholt auftritt oder du dich unwohl fühlst, solltest du mit deinem Arzt sprechen.
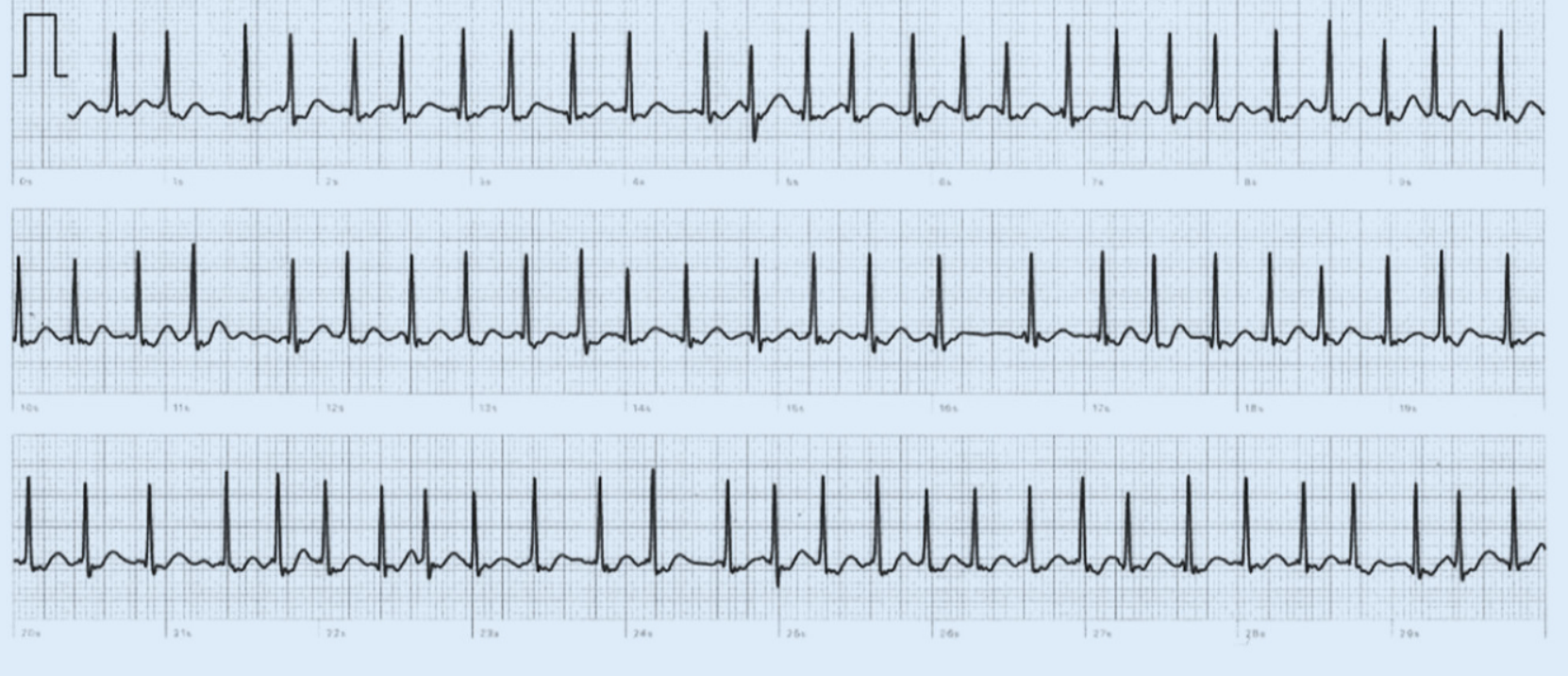

Abb. $2 \Delta$ Derselbe Patient wie in 0 Abb. 1. Weiterhin Vorhofflimmern, Kammerfrequenz 161/min. Da die Frequenz 150/min überschreitet, prüft die EKG-App nicht auf das Vorliegen von Vorhofflimmern. Apple Watch Series 5

bei EKG-Dokumentationen mit hohen Frequenzen (über 120 bzw. 150/min), die aufgrund der internen Algorithmen nicht automatisch auf Vorhofflimmern geprüft werden, vermehrt mit falschnegativen Befunden gerechnet werden muss. Eine zukünftige Optimierung der Algorithmen durch den Einsatz von künstlicher Intelligenz ist denkbar [11, 12]. Eine genaue Kenntnis der Smartwatch-spezifischen Algorithmen wird besonders dann wichtig, wenn ärztlicherseits, basierend auf den Befunden, Entscheidungen getroffen werden.

\section{Fazit}

- Smartwatch-EKGs stellen eine willkommene Erweiterung des Repertoires an diagnostischen EKGVerfahren dar.

- Mittels Smartwatch dokumentiertes Vorhofflimmern ist diagnostisch verwertbar, sofern die Diagnose von einem Arzt bestätigt wurde.

- Aufgrund diagnostischer Lücken ist ein Ausschluss von Vorhofflimmern, wie auch bei anderen EKG-Verfahren, nicht möglich.

- Beim Einsatz EKG-fähiger Smartwatches in klinischen Studien muss klar sein, dass die Häufigkeit von Vorhofflimmern unterschätzt wird.

- Es ist anzunehmen, dass sich zukünftig, z. B. basierend auf künstlicher Intelligenz, eine verbesserte diagnostische Genauigkeit ergeben wird.

\section{Korrespondenzadresse}

\section{Prof. Dr. med. Wilhelm Haverkamp}

Abteilung für Kardiologie und Metabolismus, Med. Klinik für Kardiologie, Campus VirchowKlinikum, Charité - Universitätsmedizin Berlin Augustenburger Platz 1, 13353 Berlin, Deutschland

wilhelm.haverkamp@charite.de

Funding. Open Access funding enabled and organized by Projekt DEAL.

Interessenkonflikt. W. Haverkamp, O. Göing, M. Anker und S.D. Anker geben an, dass kein Interessenkonflikt besteht.
Open Access. Dieser Artikel wird unter der Creative Commons Namensnennung 4.0 International Lizenz veröffentlicht, welche die Nutzung, Vervielfältigung, Bearbeitung, Verbreitung und Wiedergabe in jeglichem Medium und Format erlaubt, sofern Sie den/die ursprünglichen Autor(en) und die Quelle ordnungsge mäß nennen, einen Link zur Creative Commons Lizenz beifügen und angeben, ob Änderungen vorgenommen wurden.

Die in diesem Artikel enthaltenen Bilder und sonstiges Drittmaterial unterliegen ebenfalls der genannten Creative Commons Lizenz, sofern sich aus der Abbildungslegende nichts anderes ergibt. Sofern das betreffende Material nicht unter der genannten Creative Commons Lizenz steht und die betreffende Handlung nicht nach gesetzlichen Vorschriften erlaubt ist, ist für die oben aufgeführten Weiterverwendungen des Materials die Einwilligung des jeweiligen Rechteinhabers einzuholen.

Weitere Details zur Lizenz entnehmen Sie bitte der Lizenzinformation auf http://creativecommons.org/ licenses/by/4.0/deed.de.

\section{Literatur}

1. Hindricks G, Potpara T, Dagres N et al (2020) ESC guidelines for the diagnosis and management of atrial fibrillation developed in collaboration with the European Association of Cardio-Thoracic Surgery (EACTS). Eur Heart J 2020:ehaa612 


\section{Kurzbeiträge}

2. FDA (2018) De novo classification request for ECG app. https://www.accessdata.fda.gov/cdrh_docs/ reviews/DEN180044.pdf. Zugegriffen: 12. März 2020

3. FDA Electrocardiograph software for over-thecounter use. http://www.accessdata.fda.gov/ cdrh_docs/pdf20/K201525.pdf. Zugegriffen: 12. März2020

4. FDA Electrocardiograph software for over-thecounter use. http://www.accessdata.fda.gov/ cdrh_docs/pdf20/K200948.pdf. Zugegriffen: 12. März 2020

5. FDA Electrocardiograph software for over-thecounter use. https://www.accessdata.fda.gov/ cdrh_docs/pdf20/K201168.pdf. Zugegriffen: 12 März 2020

6. Withings. https://support.withings.com/ hc/de/articles/360012786977-ScanWatchBenutzerhandbuch.Zugegriffen: 12. März 2020

7. Apple (2020) Verwendung der Apple Watch zur Arrhythmieerkennung. https://www.apple.com/de/ healthcare/docs/site/Apple_Watch_Arrhythmia_ Detection.pdf.Zugegriffen: 12. März 2020

8. Jaakkola J, Hartikainen JE, Kiviniemi T et al (2015) Ventricular rate during acute atrial fibrillation and outcome of electrical cardioversion: the FinCV study. Ann Med 47:341-345

9. Perez MV, MahaffeyKW, Hedlin Hetal (2019) Largescale assessment of a smartwatch to identify atrial fibrillation. NEngl J Med 381:1909-1917

10. Dörr M, Nohturfft V, Brasier N et al. The WATCH AF Trial (2019) SmartWATCHes for detection of atrial fibrillation. JACC Clin Electrophysiol 5:199-208

11. Tison GH, Sanchez JM, Ballinger B et al (2018) Passive detection of atrial fibrillation using a commercially available Smartwatch. JAMA Cardiol 3:409-416

12. Wasserlauf J, You C, Patel Ret al (2019) Smartwatch performance for the detection and quantification of atrial fibrillation. Circ Arrhythm Electrophysiol 12:e6834

\section{Hier steht eine Anzeige.}

\section{算 Springer}

